\begin{tabular}{|c|c|}
\hline Title & A n approach to nano-chemical analysis through NC-A FM technique \\
\hline Author(s) & Suzuki, S.; Koike, Y.; Fujikawa, K.; Matsudaira, N.; Nakamura, M.; Chun, W.-J.; Nomura, M.; A sakura, K. \\
\hline Citation & $\begin{array}{l}\text { Catalysis T oday, 117(1-3), 80-83 } \\
\text { https://doi.org/10.1016/.cattod.2006.05.008 }\end{array}$ \\
\hline Issue Date & $2006-09-30$ \\
\hline Doc URL & http://hdl .handle.net/2115/15417 \\
\hline Type & article (author version) \\
\hline File Information & 5WCOC_OA 105szk_hokudai_revBW.pdf \\
\hline
\end{tabular}

Instructions for use 


\title{
An Approach to Nano-Chemical Analysis through NC-AFM Technique
}

\author{
S. Suzuki ${ }^{1)}$, Y. Koike ${ }^{1)}$, K. Fujikawa ${ }^{1)}$, N, Matsudaira ${ }^{1)}$, M. Nakamura ${ }^{1)}$, \\ W.-J. Chun ${ }^{1,2)}$, M.Nomura ${ }^{3)}$ and K. Asakura ${ }^{1) *}$
}

${ }^{1}$ Catalysis Research Center and Department of Quantum Science and Technology, Hokkaido University, 21-10 Kita, Kita-ku, Sapporo 001-0021,Japan

${ }^{2}$ Japan Science and Technology Agency, Core Research for Evolutional Science and Technology

${ }^{3}$ Photon Factory, Institute of Material Structure Science, High-Energy Accelerator Organization, Oho 1-1, Tsukuba 305-0801, Japan

\begin{abstract}
We have measured the NC-AFM frequency shift dependence on the X-ray energy around the $\mathrm{Au} \mathrm{L}_{3}$ absorption edge energy. We found a peak in the frequency shift just above the Au region at the $\mathrm{Au} \mathrm{L}_{3}$ absorption edge energy while we could not detect any peak in the frequency shift when the NC-AFM tip was placed above the Si regions. This novel phenomenon indicated that the combination of energy-variable X-rays and NC-AFM provides us a new way to nano level chemical mapping at surface. We briefly discussed some possible mechanisms.
\end{abstract}

Keyword

XANAM, NC-AFM, oxide surface

* All correspond should be addressed, email askr@cat.hokudai.ac.jp 


\section{Introduction}

Recent developments in SPM (Scanning Probe Microscopy) have provided us a clear atomic scale image of the surface. STM (Scanning tunneling microscopy) and AFM (Atomic force microscopy) are the typical examples of the SPM family. Oxide surfaces like $\mathrm{TiO}_{2}$ are successfully visualized by STM and catalytic processes on those surfaces are investigated well. However, STM experiment requires an electric conductivity because it uses a tunneling current to observe sample surfaces. In order to apply SPM to many other oxide surfaces which have little electric conductivity, AFM is another choice, but is not an easy technique because of the lack of the simplicity on the distance dependency of the atomic force between an AFM tip apex and a surface, compared to the tunneling current in the STM experiments. The AFM tip is attached to a cantilever beam which is deflected by the force acting on the tip. When the tip apex contact to the sample surface and scans laterally, the tip feels repulsive force from the surface and consequently surface roughness is imaged according to the cantilever deflection. However, the direct contact between the tip apex and the surface sometimes damages heavily both structures during the measurements. Thus AFM with the direct contact is difficult to image real atomic arrangements of the surface.

NC-AFM (Non-contact AFM) or DFM (Dynamic force microscopy) is a new device to detect much weaker attractive interaction.[1] In the case of NC-AFM, the AFM tip approaches the surface closely but does not contact to the surface directly. The tip feels an attractive force which is much weaker than the repulsive force. The cantilever oscillates at the near resonant frequency with a certain oscillation amplitude. When the attractive force changes during the scanning of the tip on the surface, the frequency of the cantilever shifts accordingly. Since the frequency shift can be measured very precisely, one may follow the surface roughness. The tip apex is just hovering or keeps a distance with negligible contact to the sample surface, which prevents structural damage like contact AFM. Since the first atomic scale image of Si was reported by Gissible and by Kitamura in 1995[2], atomic scale images of oxide surfaces like $\mathrm{NiO}, \mathrm{MgO}, \mathrm{TiO}_{2}, \mathrm{CeO}_{2}$ and $\mathrm{MoO}_{3}$ have been reported by a NC-AFM method.[3-7] However, one disadvantage for NC-AFM still remains. It is difficult to obtain elemental information directly from the NC-AFM data. Elemental analysis at sample surface is often carried out by XPS (X-ray photoelectron spectroscopy) where $\mathrm{X}$-ray excites inner shell electrons to the vacuum state as photoelectrons with specific kinetic energies. By analyzing the kinetic energy, we can know the elemental information on the sample surface.

If XPS is combined with electron microscopy, one can have a microscopic chemical 
imaging of the surface. EXPEEM (Energy selected photoemission electron microscopy) is an example for this combination and nano-meter scale surface images have been successfully obtained.[8-11] If the combination of NC-AFM and XPS is possible, we can have an atomic scale elemental analysis method.

But is it possible to control the atomic force by X-ray? First we should think about the origin of the force between a tip apex and the surface in the NC-AFM. One may think of van der Waals force or electrostatic force as the main origin for atomic force. Recent theoretical and experimental investigations of NC-AFM revealed that the covalent chemical bond force was contained as the effective force component for the imaging at the atomic level. Therefore, if one may change electron density of the covalent bond between the tip and the surface, one can control the covalent bond strength and modify the atomic force between them. In this paper we presented one example to show the possibilities to modify the atomic force between the tip apex and the sample surface by X-ray irradiation during NC-AFM measurements. The data were obtained at several points on $\mathrm{Au}$ and Si regions to confirm the reproducibility of this phenomenon and we have discussed its mechanisms.

\section{Experimental}

Figure 1 shows our instrument to measure the atomic force as a function of X-ray energy. All measurements were carried out at the Photon Factory in Japan. The system consisted of two chambers; an AFM chamber and a pre-treatment chamber. The base pressures for both chambers were $10^{-8} \mathrm{~Pa}$. The spatial resolution of the NC-AFM microscope was less than $10 \mathrm{~nm}$. There were two Be windows equipped on the AFM chamber for X-ray entrance and exit. A sample was mounted on a stage with three axes and a rotation mechanism. We could look inside the chamber through a CCD camera to adjust the sample position relative to X-ray beam. Figure 2 shows a CCD picture with the X-ray beam coming from the right side and hitting the sample surface. In front of the sample, we placed phosphor screens to confirm the X-ray position. A $\mathrm{Si}$ wafer with square $\mathrm{Au}$ islands in a period of $25 \mu \mathrm{m}$ was used for a test

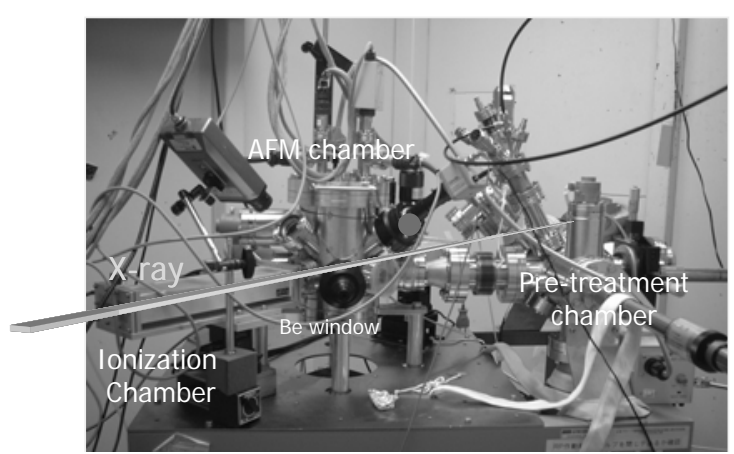

Fig.1 a photograph of a NC-AFM chamber installed in the $\mathrm{X}$-ray protective hatch at the beam line at Photon Factory

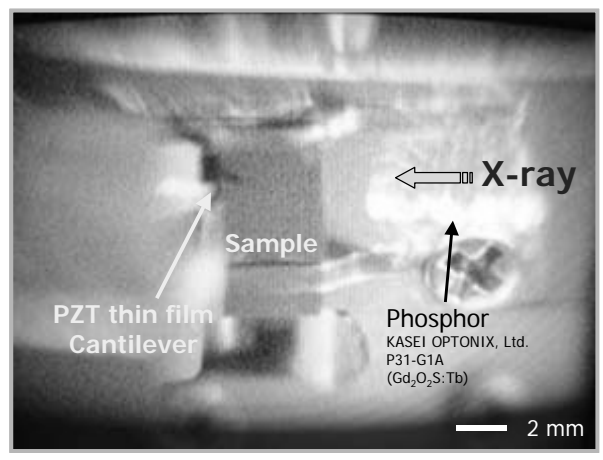

Fig.2 a photograph inside the chamber around the AFM stage. 
sample. The thickness and width of the Au square islands were $20 \mathrm{~nm}$ and $10 \mu \mathrm{m}$, respectively.

\section{Results}

Figure 3 shows a NC-AFM image of the sample surface. A white region is the $\mathrm{Au}$ island and a dark region is the $\mathrm{Si}$ substrate. The frequency shift measurements were carried out as follows: First, move the cantilever above the certain region. Second, stop the feedback loop routine and keep a distance between the tip apex and the surface constant. Third, irradiate the $\mathrm{X}$-ray to the sample surface and start to scan its energy with monitoring the cantilever deflection. We scanned the incident $\mathrm{X}$-ray energy from $-50 \mathrm{eV}$ to $50 \mathrm{eV}$ around the $\mathrm{L}_{3}$ absorption edge.

Figure 4 shows the frequency shift during the scanning of the X-ray. When we fixed the AFM tip apex above the $\mathrm{Au}$ region and measured the $\mathrm{X}$-ray energy dependency of the cantilever deflection, a frequency shift peak appeared above the $\mathrm{Au} \mathrm{L}_{3} \mathrm{X}$-ray absorption edge. On the other hand we could not observe any peak when the tip was fixed on the $\mathrm{Si}$ region. We moved the NC-AFM tip all around the sample surface and repeated the experiments on

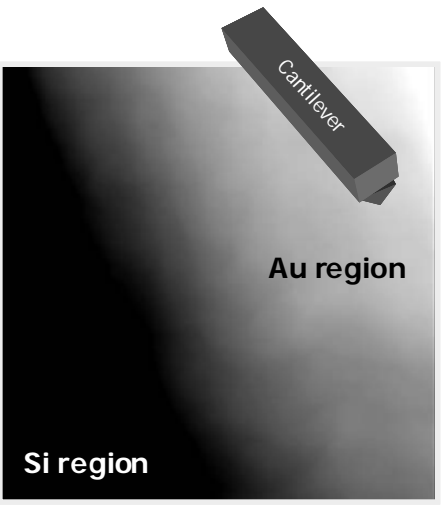

Fig.3 a NC-AFM image of a Au/Si sample. $3.5 \times 3.5 \mu \mathrm{m}^{2}$,

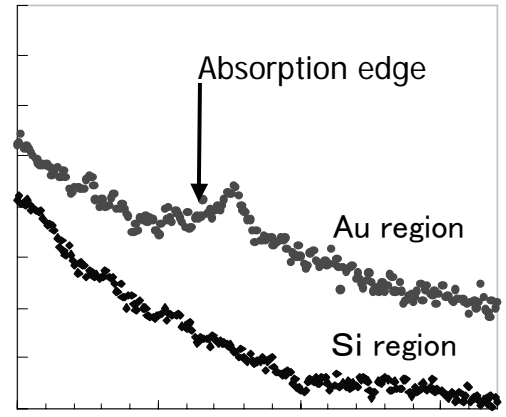

Fig.4 Frequency shift spectra normalized by $\mathrm{I}_{0}$ on both Au and Si regions different regions. Figure 5 shows bundles of spectra

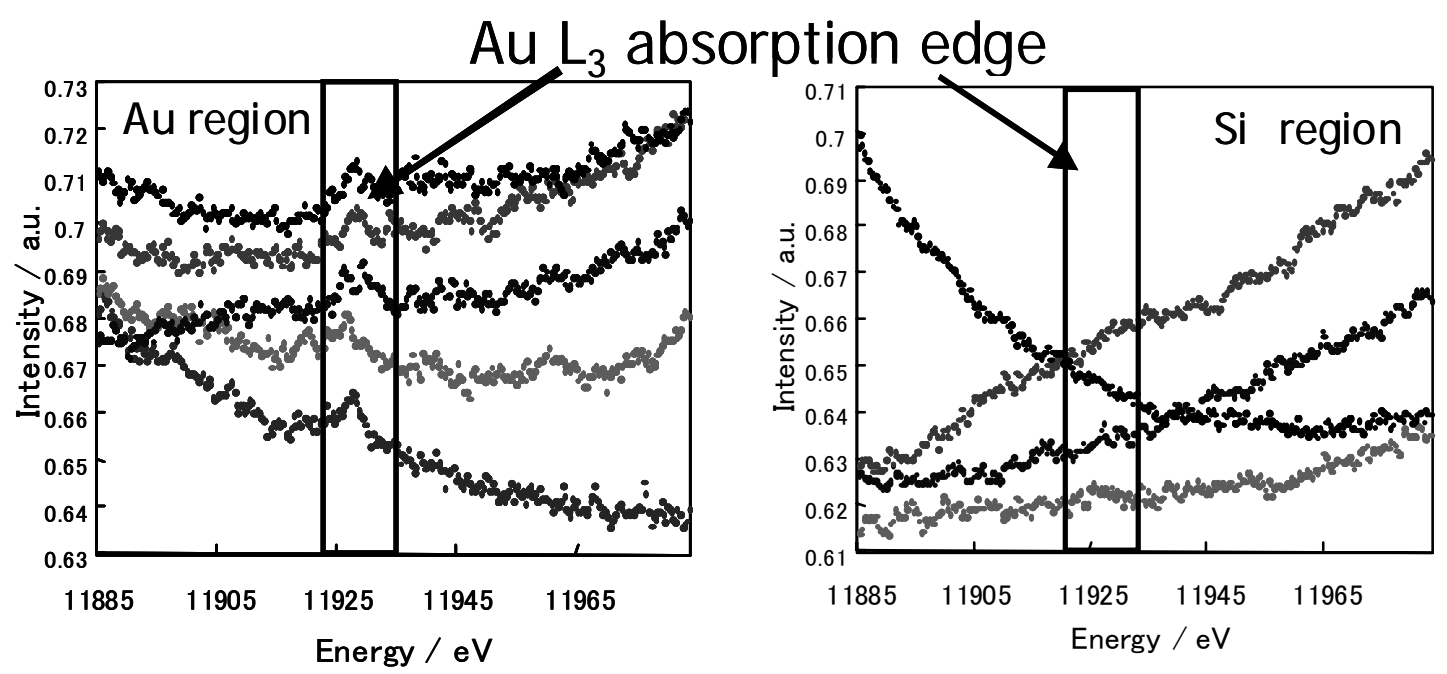

Fig.5 Frequency shift spectra at various sites on the Au and Si regions 
on several Au and Si regions. The peaks always appeared just at the $\mathrm{Au}_{3}$ absorption edge when the tip was fixed above the Au regions, while no peak was observed above the Si region. This demonstrated the possibility to modify the cantilever deflection by means of X-ray with its energy around the absorption edge, which leads to a new method to identify the element by NC-AFM measurements.

\section{Discussion}

We originally thought the frequency shift occurred due to the electron transition to the unoccupied state of the bonding orbital between the tip and the surface. We should take other possibilities into account.

1. Thermal effect induced by X-ray absorption

2. Charge effect induced by X-ray absorption

3. X-ray localization in the nano-space between the tip apex and surface at the absorption energy.

The first two possibilities can be denied because the frequency shift followed the absorption spectrum of $\mathrm{Au} \mathrm{L}_{3}$ and absorption edge like shape should appear if the spectrum change occurred due to the thermal effect or the charge effect. Hence, the third candidate is another possible mechanism. As is found in SERS (Surface enhanced Raman spectroscopy), electric field of X-ray can be concentrated around the atomically sharp tip apex. If the tip apex is approached closely to the sample surface in the order of X-ray wavelength(sub-nano meter), the strong electric field may be localized between them and the X-ray absorption may be resonantly enhanced. Although we need further studies by changing the tip distance and bias voltage to confirm the real mechanisms, this novel phenomenon may lead to an atomic scale technique called XANAM (X-ray aided non-contact atomic force microscopy)[12].

\section{Acknowledgement}

This work has been supported by Grand-in-Aid for Scientific Research for young scientist (B) (2004-2005), CREST project "Nano Environmental Catalysis" of JST. The work has been carried out under the approval of Photon Factory Advisory Committee (Proposal number 2003G301).

\section{References}

[1] S. Morita, R. Wiesendanger, E. Meyer (Eds.), Non-contact Atomic Force Microscopy, Berlin, Springer, 2002.

[2] F.J. Gissible, Science 267 (1995) 68; S. Kitamura, M. Iwatsuki, Jap. J. Appl. Phys.34 (1995) L145.

[3] H. Hosoi, K. Sueoka, K. Hayakawa, K. Mukasa, Applied Surf.Sci. 157 (2000) 
218.

[4] K. Fukui, Y. Iwasawa, Surf. Sci., 441 (1999) 529.

[5] K. Fukui, H. Onishi, Y. Iwasawa, Phys.Rev.Lett. 79 (1997) 4202.

[6] K. Fukui, Y. Namai, Y. Iwasawa, Appl. Surf.Sci., 188 (2002) 252.

[7] S. Suzuki, Y. Ohminami, T. Tsutsumi, M.M. Shoaib, M. Ichikawa, K. Asakura, Chem.Lett. 32 (2003) 1098.

[8] R. Wichtendahl, R. Fink, H. Kuhlenbeck, D. Preikzsas, H. Rose, R. Spehr, P. Hartel, W. Engel, R. Schlögl, H.-J. Freund, A.M. Bradshaw, G. Lilienkamp, Th. Schmidt, G. Benner, and E. Umbach, Surf. Rev. Lett. 5 (1998) 1249.

[9] H. Yasufuku, Y. Ohminami, T. Tsutsumi, K. Asakura, M. Kato, Y. Sakai, Y. Kitajima, Y. Iwasawa, Chem.Lett. (2002) 842.

[10] H. Niimi, T. Tsutsumi, H. Matsudaira, T. Kawasaki, S. Suzuki, W.J. Chun, M. Kato, Y. Kitajima, Y. Iwasawa, K. Asakura, Appl.Surf.Sci. 237 (2004) 641.

[11] H. Yasufuku, Y. Ohminami, T. Tsutsumi, H. Niimi, N. Matsudaira, K. Asakura, M. Kato, Y. Sakai, Y. Kitajima, Y. Iwasawa, Jpn.J.Appl.Phys. 43 (2004) 7682.

[12] S. Suzuki, Y. Koike, K. Fujikawa, W.-J. Chun, M. Nomura, K. Asakura, Chem.Lett. 33 (2004) 636. 